\title{
Overweight and obesity in preschool children in Turkey: A multilevel analysis
}

\author{
Şeyma Görçin Karaketir $\mathrm{MD}^{1 \star \mathbb{D}}$, Nimet Emel Lüleci $\mathrm{MD}^{2}$ (D), Mehmet Ali Eryurt $\mathrm{PhD}^{3}$ (D), \\ Ahmet Naci Emecen ${ }^{4}$ (D) Mehmet Haklıdır ${ }^{5}$ (D) and Seyhan Hıdıroğlu PhD $^{6}$ (D)
}

${ }^{1}$ Department of Public Health, Occupational Health Training Programme, Istanbul University, Istanbul School of Medicine, Istanbul, Turkey, ${ }^{2}$ Department of Public Health, School of Medicine, Marmara University, Istanbul, Turkey, ${ }^{3}$ Institute of Population Studies, Hacettepe University, Ankara, Turkey, ${ }^{4}$ Department of Public Health, Epidemiology Subsection, Dokuz Eylul University School of Medicine, Izmir, Turkey, ${ }^{5}$ TUBITAK BILGEM, Gebze, Kocaeli, Turkey and ${ }^{6}$ Department of Public Health, School of Medicine, Marmara University, Istanbul, Turkey

*Corresponding author. Email: gorcin_eseyma@hotmail.com

(Received 17 April 2021; revised 2 January 2022; accepted 5 January 2022; first published online 28 January 2022)

\begin{abstract}
Childhood obesity/overweight is a worldwide concern and its prevalence is increasing in many countries. The first aim of this study is to analyse the trends in overweight and obesity among children under the age of five in Turkey based on the new World Health Organization (WHO) standards, using data from the 'five-round of the Turkey Demographic and Health Surveys' (TDHSs). The second aim is to examine whether or not the maternal/household and individual-level factors are associated with overweight/obesity using TDHS 2003, 2008, and 2013 datasets. A total sample of 14,231 children under the age of five were extracted from the TDHS in 1993, 1998, 2003, 2008, and 2013 to determine the prevalence of the trend. Pooled data from 8,812 children were included in the analysis to examine factors associated with overweight/obesity. Taking into account the clustered data structure, multilevel logistic regression models were utilised. In 1993, 1998, 2003, 2008, and 2013 the prevalence of overweight children was 5.3\%, 4.9\%, 10.0\%, $11 \%$ and $11.6 \%$, respectively. The factors that were independently associated with overweight/obesity were as follows: living in single-parent households (adjusted odds ratio $(\mathrm{aOR})=2.27,95 \% \mathrm{CI}=1.21$ 4.26), compared to living in dual-parent households; having an obese mother ( $\mathrm{aOR}=4.25,95 \%$ $\mathrm{CI}=1.73-10.44)$, overweight mother $(\mathrm{aOR}=3.15,95 \% \mathrm{CI}=1.29-7.69)$, and a normal-weight mother $(\mathrm{aOR}=2.70,95 \% \mathrm{CI}=1.11-6.59)$ compared to having an underweight mother; being aged between 13-24 months $(\mathrm{aOR}=1.72,95 \% \mathrm{CI}=1.30$ to 2.27$)$, compared to being aged $0-12$ months; male gender $(\mathrm{aOR}=1.30,95 \% \mathrm{CI}=1.11$ to 1.53$)$; being stunted $(\mathrm{aOR}=2.18,95 \% \mathrm{CI}=1.74$ to 2.73$)$; high birth weight $(\mathrm{aOR}=1.55,95 \% \mathrm{CI}=1.08$ to 2.23$)$ compared to low birth weight. In addition, overweight was higher in children of mothers who had completed primary school ( $\mathrm{aOR}=1.21,95 \% \mathrm{CI}=1.01$ to 1.59$)$ than children of mothers who had not completed primary school. These findings reveal that, over the years, there has been a substantial increase in obesity/overweight among children which demonstrates the importance of evaluating the overweight indicators at the maternal/household level.
\end{abstract}

Keywords: Child Health and Nutrition; Obesity; Maternal and Child Health

\section{Introduction}

Overweight and obesity are the major risk factors for many chronic diseases, particularly for diabetes, cardiovascular diseases, and cancer (WHO, 2020a). These diseases, namely noncommunicable diseases (NCDs), are not only the cause of premature mortality, but also of 
long-lasting morbidity. Moreover, overweight/obesity in children is linked with impairments in quality of life (Tsiros et al., 2009; Williams et al., 2005) and a high risk of teasing and bullying (Lobstein et al., 2004). One of the major concerns on the subject is that childhood overweight/ obesity is likely to continue throughout the adulthood of the individual (Rooney et al., 2011). Thus, the increasing prevalence of obesity poses a significant threat to future demands for healthcare. Eventually, prevention of childhood obesity becomes a priority for reducing the financial and general burden on healthcare.

The prevalence of childhood overweight/obesity has increased significantly in most highincome countries, and is also increasing rapidly in low- and middle-income countries (Sahoo et al., 2015). WHO reported (2020b) that 39 million children under the age of five worldwide were overweight in 2019. The number of overweight preschool children has increased by almost 24\% since the year 2000 in Africa. And approximately half of the children under five who were overweight/obese in 2019 lived in Asia (WHO, 2020b). As such, halting the rise in diabetes and obesity in children and adults became one of the global health targets set by the World Health Assembly in 2013 (WHO, 2013). Improving nutrition occupies a substantial place in the Sustainable Development Goals (SDG) for 2015-2030 (WHO, 2016).

In the SDG framework, it is stated that improving nutrition plays a key role in health, education, empowerment of women, employment, progress in poverty, and inequality of a society. Issues such as: poverty and inequality, water, sanitation and hygiene, education, food systems, climate change, social protection, and agriculture have also been reported to have significant effects on the outcomes of nutrition (United Nations, 2015). Economic, genetic, cultural, behavioural, and psychosocial factors are related to excess weight, making this a complicated and multifaceted context (Franks and Ling, 2010; Pérez et al., 2013). To determine the most powerful strategies for reducing the burden of overweight/obesity and sustainable development, the prevalence and determinants of overweight/obesity must be known (Mohsena et al., 2016). WHO highlights the importance of monitoring levels and trends of overweight/obesity in children (De Onis et al., 2010). However, in Turkey, preschool childhood obesity is not yet perceived as an emerging health issue and receives inadequate attention and the data related to overweight/obesity trends among preschool children is inadequate. Few studies that have been conducted are cross-sectional, and the data are limited to one year (MOH/HUBDB/NH, 2010; Santas \& Santas, 2018). There is a need to conduct studies using world-standard anthropometric measures and shed light on factors associated with overweight/obesity in preschool children in Turkey. This study is one of the first studies to use world-standard anthropometric measures in Turkey with the following objectives: firstly, to describe the prevalence and trends of overweight/obesity among preschool children based on population body mass index-for-age Z-score (BMI-for-age), which is the globally preferred anthropometric measure instead of weight-for-length and -height or weight-for-age. In this way, the worldwide data would be comparable with Turkey. Secondly, to identify the 25 -year trends of overweight/obesity in preschool children in Turkey using the recent 2006 WHO standards instead of the National Center for Health Statistics (NCHS) reference. And lastly, to examine whether the maternal/household and individual-level factors are associated with overweight/ obesity in preschool children using TDHS 2003, 2008, and 2013 datasets.

\section{Methods}

\section{Data source}

Demographic and Health Surveys (DHSs) are regularly carried out in over 90 countries worldwide and provide for the major basic demographic indicators and the health status in those countries to be measured by validated questionnaires. DHS uses a weighted, multi-stage, stratified cluster sampling methodology that represents the general population. In this study, the data provides the prevalence and trends of overweight and obesity among preschool children, and is obtained from 


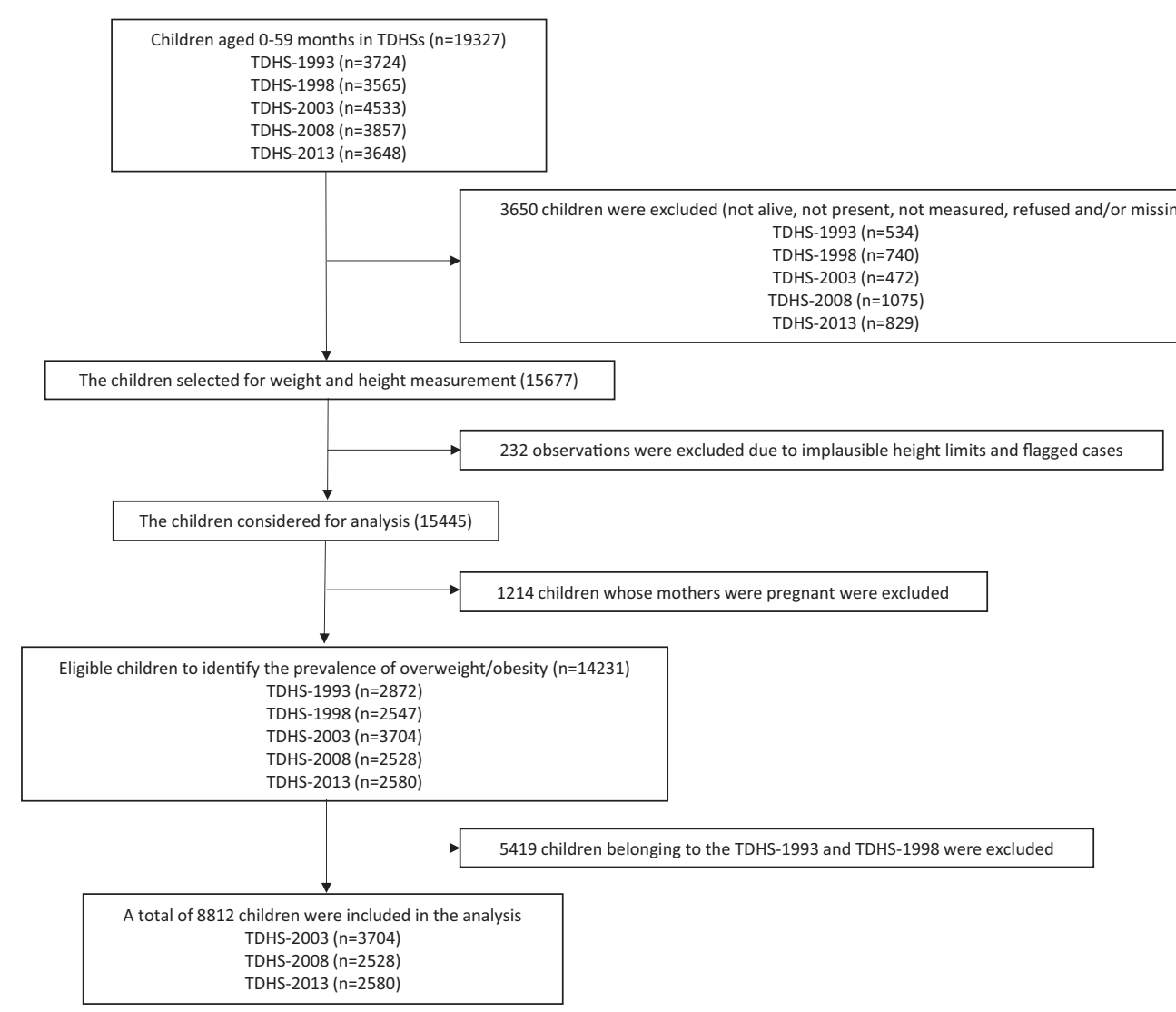

Figure 1. The study flowchart of the selection of the final study population from the TDHS datasets.

the last five TDHSs conducted by the Hacettepe University Institute of Population Studies (HIPS) in $1993,1998,2003,2008$, and 2013. To examine whether the maternal/household and individuallevel factors are associated with overweight/obesity in preschool children, a pooled data of TDHS 2003, 2008, and 2013 is utilized. The prevalence of overweight/obesity was doubled beginning from TDHS 2003 when compared to the previous two TDHSs. The main reasons why only the last three TDHS studies were used are; a) it is possible to get more feasible results with contemporary data, b) to add comparability to the results of this study under a high-prevalence overweight/obesity setting. Moreover, a larger set of data is obtained by pooling the last three surveys which led to the increased number of observations and more reliable estimations. More details about TDHS surveys are available in the survey reports (HIPS, 2020).

The dataset included information from children under five years old and their mothers aged between 15 and 49 years old. Children with no anthropometric measurements or who have had implausible measurements were excluded. The children with pregnant mothers were also excluded because variables such as maternal BMI and maternal employment status were affected by pregnancy and could mislead the results. Among the total of 14,231 eligible children to identify the overweight/obesity prevalence, the contribution of the TDHS 1993, 1998, 2003, 2008, and 2013 was 2872, 2547, 3704, 2528, and 2580 children, respectively. When the TDHS 1993 and TDHS 1998 were excluded, the pooled data of 8,812 children under five years old were included in the analysis to examine the factors associated with overweight/ obesity (Figure 1). 


\section{Anthropometric measurements and outcome variables}

Weight and height/length were measured by trained staff contracted by the DHS survey, using standardized measuring equipment. The supine length was measured for children below 24 months and standing height was measured for children over 24 months. Details on anthropometric measurement in DHS can be found in (ICF International, 2012). Overweight was defined as body mass index-for-age more than 2 standard deviations (SD) above the median of the reference population (BMI-for-age $>+2$ ) and obesity was defined as BMI-for-age more than 3 SD. Children with BMI-for-age Z-score above 2 SD were categorized as overweight/obese and $2 \mathrm{SD}$ or below as normal. Implausible values beyond $+/-5 \mathrm{SD}$ were excluded based on recommendations by WHO (2019a).

\section{Multicentre Growth Reference Study and 2006 WHO Child Growth Standards}

Standard implementation for examining anthropometric data is the comparison of the population studied with a reference population based on the properties of the normal distribution. It enables to compare the changes between the anthropometric indicators of different groups in the community and the changes that may occur in nutritional status over time. It is worth mentioning that the reason for using the reference group is that well-nourished children in all communities follow almost the same growth patterns before puberty (HIPS, 2014).

In 2006, the WHO released the most recent WHO Child Growth Standards (WHO Multicentre Growth Reference Study Group \& De Onis, 2006) based on a multi-country study involving breastfed infants and young children from 6 geographically distinct countries (Brazil, Ghana, India, Norway, Oman, and the United States). However, in TDHS-1993, TDHS-1998, and TDHS-2003 databases, the analyses were based on estimates derived from NCHS growth references (Hamill et al., 1977), which were recommended for international use. But the recent WHO Child Growth Standards differ substantially from the NCHS reference, therefore, the NCHS and recent WHO standards are not comparable and compatible (De Onis et al., 2006). This study aimed to describe the national prevalence and trends of early childhood overweight/obesity using the recent WHO Child Growth Standards because these standards provide better statistical depth and lead to more robust information out of data in hand. Thus, Z-scores and the prevalence of overweight for 1993, 1998, and 2003 databases were computed using a syntax file provided by WHO (2006). DHS data experts at www.dhsprogram.com were also contacted and requested anthropometric indicators for TDHS 1993, 1998, and 2003 calculated according to the recent WHO standards. Either way, the obtained BMI-for-age indicators gave the same results. Thus, the BMI-for-age from 1993 to 2013 became comparable at both national and global levels.

\section{Independent variables}

Independent variables were identified using the standard questionnaires and the DHS recoding manual (ICF International, 2018). Children, mothers, and household characteristics that were considered to be relevant based on the literature were chosen amongst the existing variables in the databases.

Household and maternal characteristics: Region comprised five categories: North, West, Middle, East, and South. Place of residence was urban or rural. Wealth index quintile, a variable indicating an economic well-being score based on housing characteristics and ownership of sustainable goods (Rutstein and Kiersten, 2004), initially comprised five quintiles: poorest, poorer, middle, richer, richest. For mother's marital status, the initial categories "married" and "living with a partner" were regrouped into "couple" category; the other initial categories ("never in union", "widowed", "divorced" and "separated") were put together in the "single parent" category. Mother's age, initially a quantitative variable was recoded into three categories: 15-19, 20-29, $\geq 30$ years. Mother's BMI, initially a quantitative variable was recoded into four categories, based 
on the international classification of adult overweight and obesity: underweight $\left(<18.5 \mathrm{~kg} / \mathrm{m}^{2}\right)$, normal weight $\left(18.5-24.9 \mathrm{~kg} / \mathrm{m}^{2}\right)$, overweight $\left(25-29.9 \mathrm{~kg} / \mathrm{m}^{2}\right)$ and obese $\left(\geq 30 \mathrm{~kg} / \mathrm{m}^{2}\right)$. Weight and height were measured by trained staff, using standardized measuring equipment. Mother's mother tongue the initial categories were "Turkish" and "Kurdish"; the other categories (mainly "Arabic" and other languages) were put together in the "Other" category. Mother's education and father's education comprised four categories: no/incomplete primary school, primary school, secondary school, high school, and higher. Mother's employment status comprised three categories: not employed, employed without social security, employed with social security.

Child characteristics: Age of the child was recoded in months' groups: 0-11, 12-23, 24-35, 36-59 months. Sex of the child was female and male. Type of birth was normal and caesarean. Duration of breastfeeding, initially a quantitative variable, was recoded into three categories: the child is less than 6 months old and is still breastfeeding, $<6$ months, $\geq 6$ months. Bottle-feeding derived from drank from the bottle with a nipple yesterday/last night variable and comprised two categories: yes, and no. Birth weight was categorized into three groups, based on clinical cut-off points of birth weight: low $(<2500 \mathrm{~g})$, middle $(2500 \mathrm{~g}-4000 \mathrm{~g})$, and high birth weight $(>4000 \mathrm{~g})$. Stunting was defined as height-for-age less than 2 SD below the median of the reference population. Extreme values beyond +/- $6 \mathrm{SD}$ were excluded according to recommendations by WHO (2010). Therefore, stunted, initially a quantitative variable, was recoded into two categories: yes, and no.

\section{Data analysis}

The TDHS surveys collect data at the national level, with rural-urban distinction, and at the regional level. Detailed information on all other control variables came from the relevant modules of the survey household and individual questionnaire forms. While the sample designs and questionnaires of the surveys were the same, their sample sizes differed. Consequently, the number of women interviewed and the number of children whose information was collected through birth histories were different. Therefore, to prevent possible biases in the analyses stemming from the different number of observations in different surveys, weighting factors obtained from the equation $1 /(a \times n c / n T)$ were used (Marriott et al., 2007), where $\mathrm{a}$ is the total number of surveys, $\mathrm{n}_{\mathrm{c}}$ is the total number of respondents for the survey $c$, and $\mathrm{n}_{\mathrm{T}}$ is the total number of respondents for all surveys. Weighting factors were multiplied by sample weight to obtain a new weight variable and final weights. Using the final weights, the prevalence of overweight and obesity was adjusted and the multilevel analysis was done.

Categorical variables were compared using the chi-square test. Maternal/household and individual-level factors associated with overweight/obesity were investigated using generalized linear mixed-effects models with canonical link (logit). The group-level variable in this study was the clusters in which the sampling frame was divided; they were called primary sampling units. After the sample size per domain was determined, the sample size was converted to the number of primary sampling units or clusters that would be selected. TDHS surveys included 700, 634, and 642 clusters in 2003, 2008, and 2013, respectively. First, the null model (Model 1-random intercept model) was fitted with no predictors, considering cluster-specific random effects on being overweight/obese. It was then observed that the between-cluster variance was non-zero, and randomintercept fixed slope models were fitted by adding maternal/household and individual level explanatory variables. The second model (Model 2) included maternal/household variables, and the third model (Model 3) included individual-level variables. The final model (Model 4) was fitted for each maternal/household and individual-level variable. The survey year was fitted as a fixed effect in all multivariate models. Between-cluster effects were presented with an intraclass correlation coefficient (ICC, \%), a proportional change in variance (PCV, \%), and median odds ratios (MOR). The ICC represents the proportion of the total observed individual variation in the outcome that was attributable to between-cluster variation. It was calculated by the formula 


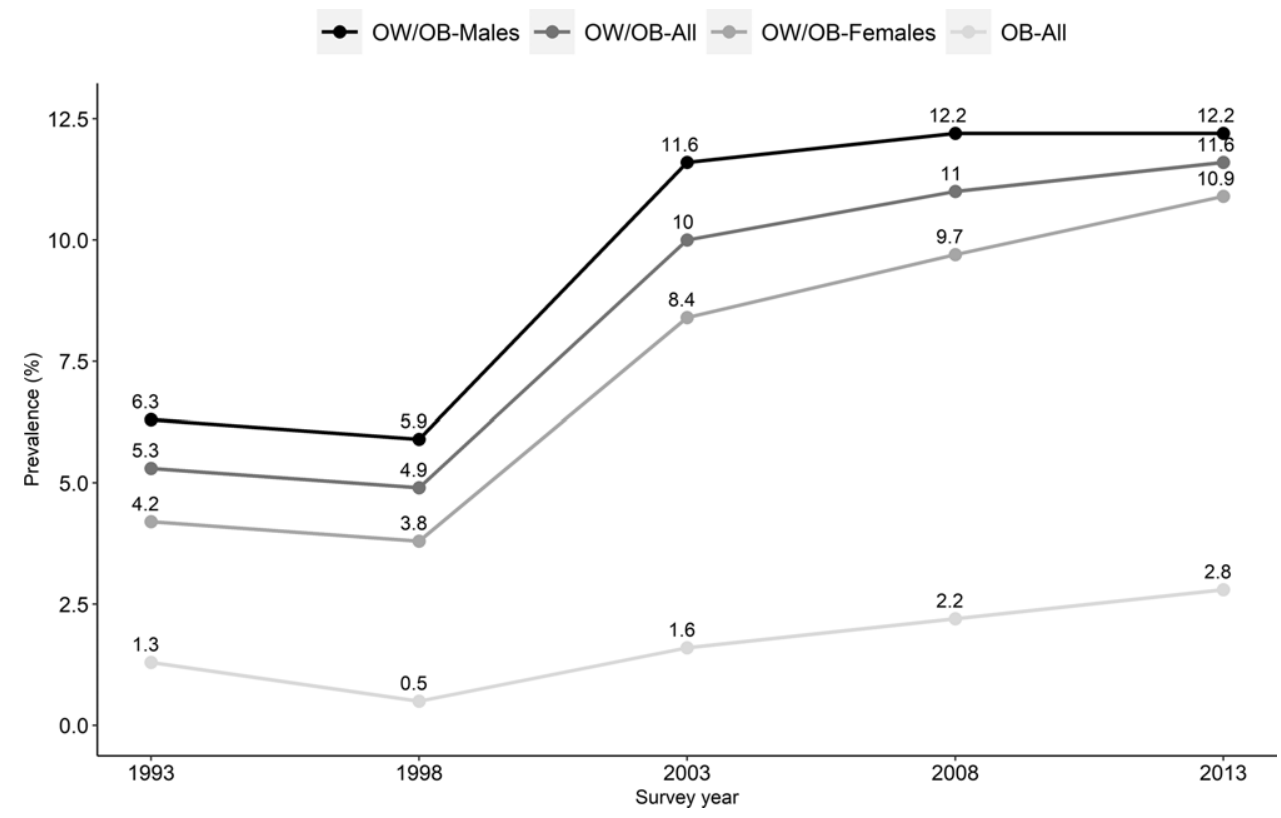

Figure 2. Trend in overweight (OW) and obesity $(\mathrm{OB})$ prevalence among children under five years, Turkey Demographic and Health Survey 1993, 1998, 2003, 2008 and 2013.

$\tau^{2} /\left(\tau^{2}+\pi^{2} / 3\right)$ where $\tau^{2}$ denotes the between-cluster variation. PCV represented the proportional change in cluster variation between the null model and model 2 , model 3 , model 4 , respectively. MOR was the median distribution of odds ratios comparing two randomly selected subjects with the same covariate patterns, but in different clusters, comparing the higher risk subject to the lower risk subject. The fixed effects of explanatory variables were provided as adjusted odds ratios (aOR) and their 95\% confidence intervals (95\% CI). Statistical analyses were performed in $\mathrm{R}$ version 3.6.1 (a language and environment for statistical computing, $\mathrm{R}$ Foundation for Statistical Computing, Vienna, Austria. URL: https://www.R-project.org) using lme4 package. The significance level was defined at the double-sided p-value of less than 0.05 .

\section{Results}

\section{Descriptive statistics}

In 1993, 1998, 2003, 2008, and 2013 the prevalence of overweight (BMI-for-age $>+2$ ) was 5.3\%, $4.9 \%, 10.0 \%, 11 \%$, and $11.6 \%$, and that of obesity (BMI-for-age $>+3$ ) $1.3 \%, 0.5 \%, 1.6 \%, 2.2 \%$, and $2.8 \%$, respectively. The prevalence of overweight in preschool children had more than doubled over 25 years and the prevalence was higher in males than females each year (Figure 2).

Figure 3, TDHS-2003, TDHS-2008, and TDHS-2013 data sets generated by the pooled data sets by region shows the prevalence of overweight children in Turkey. The highest prevalence was in West Anatolia with 13.8\%, the lowest in Central East Anatolia with 5.9\%, and the average prevalence in all regions was $10.9 \%$.

Various characteristics of preschool children are presented in Table 1. Data shows that, based on the mother's education variable, urban settlement has increased, the percentage of adolescent pregnancy has decreased; the percentage of high school and higher education graduates has increased (from $14.6 \%$ to $25.9 \%$ ). A large proportion of mothers (46.5\%) were graduates of primary school. Stunting in children decreased from $14.5 \%$ in 2003 to $9.6 \%$ in 2013. High birth weight has also decreased throughout the years (Table 1). 


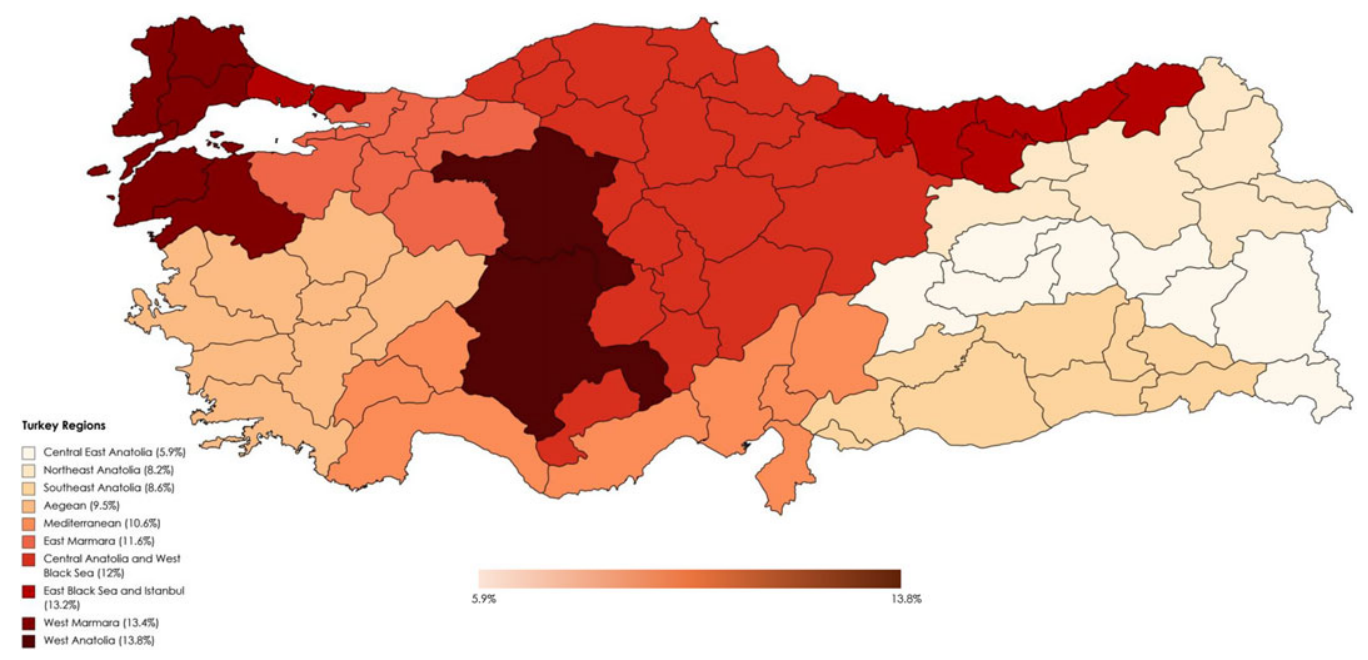

Figure 3. Prevalence of child overweight across the regions; pooled data from TDHS-2003, TDHS-2008 and TDHS-2013.

\section{Bivariate associations of outcomes with independent variables}

Table 2 shows the descriptive statistics indicating the relationship between overweight status of preschool children and household/maternal- and individual-level factors. No difference was observed in the prevalence of overweight/obesity between children from urban and rural areas. The prevalence of obesity was lowest in the poorest quintile with $9.7 \%$. The difference between the poorest and the richest quintiles (13\%) was significant; as well as the difference between the poorest and fourth quintiles (12.3\%). Around 24\% of mothers were overweight/obese. The prevalence of overweight/obesity among preschool children was the highest among children born to mothers with $\mathrm{BMI} \geq 30$. The significance was due to the difference between underweight-obese and normalobese groups. Overweight/obesity was higher in children of women whose mother tongue was Turkish (11.4\%), and women who were graduated from a high school or higher education level (12.3\%). The statistical significance was due to the difference between no/incomplete primary school and high school or higher education level. As the education level of the father increased, the overweight/obesity status of the child increased, and the significance was due to the difference between primary $(9.9 \%)$ and high school or higher education graduates $(12.3 \%)$. The prevalence was the highest in male children (12\%), children of age 13-24 months (16.3\%), and children who were bottle-fed (13\%). Also, the prevalence was higher among children who had high birth weight (15\%). The significance was due to the difference between the low-high birth weight and middlehigh birth weight groups. Stunting was also more common among overweight children ( $16 \%$ of stunted children were overweight).

\section{Results of the multilevel logistic regression models}

Table 3 shows the summary measures of between-cluster effects. The ICC derived from the null model was $15.36 \%$, which implies that $15.36 \%$ of the individual variation in the odds of childhood overweight/obesity was due to the effect of clusters, while the remaining $84.6 \%$ was due to unmeasured differences between the children. When comparing MOR with the ORs for the maternal/ household (model 2) and individual (model 3) characteristics, the median effect of clustering on childhood overweight/obesity was high. Results of the pooled-data multilevel logistic regression models were presented in Table 4. 
Table 1. Percentage and frequency distribution of the characteristics of children under five years in Turkey (Weighted)

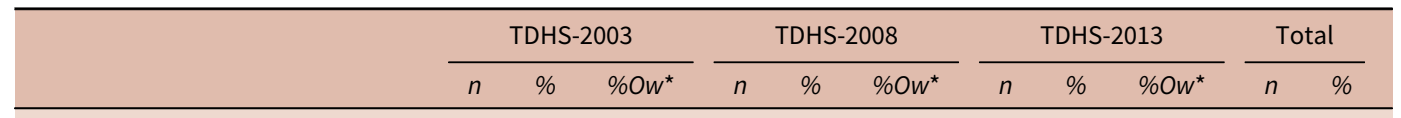

\begin{tabular}{|c|c|c|c|c|c|c|c|c|c|c|}
\hline \multicolumn{11}{|l|}{ Region } \\
\hline West & 902 & 33.4 & 9.8 & 932 & 34.7 & 12.3 & 990 & 36.9 & 14.3 & $2824 \quad 35.0$ \\
\hline South & 364 & 13.5 & 9.7 & 368 & 13.7 & 10.3 & 412 & 15.3 & 11.5 & $1145 \quad 14.2$ \\
\hline Middle & 546 & 20.2 & 9.9 & 596 & 22.2 & 12.1 & 451 & 16.8 & 15.1 & $1593 \quad 19.7$ \\
\hline North & 159 & 5.9 & 15.3 & 137 & 5.1 & 12.4 & 176 & 6.5 & 10.3 & $472 \quad 5.9$ \\
\hline East & 727 & 27.0 & 9.4 & 651 & 24.3 & 8.2 & 657 & 24.5 & 5.6 & $2035 \quad 25.2$ \\
\hline
\end{tabular}

Place of residence

\begin{tabular}{|c|c|c|c|c|c|c|c|c|c|c|c|}
\hline Rural & 892 & 33.1 & 9.8 & 731 & 27.2 & 12.5 & 566 & 21.1 & 8.3 & 2188 & 27.1 \\
\hline Urban & 1806 & 66.9 & 10.1 & 1953 & 72.8 & 10.5 & 2121 & 78.9 & 12.5 & 5880 & 72.9 \\
\hline \multicolumn{12}{|l|}{ Wealth index quintile } \\
\hline Lowest (poorest) & 639 & 23.7 & 9.8 & 636 & 23.7 & 11.0 & 590 & 22.0 & 8.2 & 1866 & 23.1 \\
\hline Second & 581 & 21.5 & 11.6 & 643 & 24.0 & 10.3 & 615 & 22.9 & 9.0 & 1838 & 22.8 \\
\hline Middle & 515 & 19.1 & 7.4 & 580 & 21.6 & 10.7 & 567 & 21.1 & 11.9 & 1662 & 20.6 \\
\hline Fourth & 555 & 20.6 & 11.7 & 437 & 16.3 & 9.4 & 477 & 17.7 & 15.7 & 1469 & 18.2 \\
\hline Highest (richest) & 408 & 15.1 & 9.2 & 387 & 14.4 & 14.5 & 438 & 16.3 & 15.2 & 1233 & 15.3 \\
\hline \multicolumn{12}{|l|}{ Marital status } \\
\hline Couple & 2661 & 98.6 & 10.1 & 2661 & 99.1 & 11.0 & 2655 & 98.8 & 11.4 & 7977 & 98.9 \\
\hline Single parent & 37 & 1.4 & 5.4 & 23 & 0.9 & 10.3 & 32 & 1.2 & 30.5 & 91 & 1.1 \\
\hline \multicolumn{12}{|l|}{ Mother's age (years) } \\
\hline $15-19$ & 85 & 3.1 & 8.4 & 59 & 2.2 & 18.0 & 53 & 2.0 & 8.8 & 197 & 2.4 \\
\hline $20-29$ & 1572 & 58.3 & 9.9 & 1517 & 56.5 & 11.8 & 1266 & 47.1 & 12.0 & 4355 & 54.0 \\
\hline$\geq 30$ & 1041 & 38.6 & 10.3 & 1108 & 41.3 & 9.6 & 1368 & 50.9 & 11.4 & 3517 & 43.6 \\
\hline \multicolumn{12}{|l|}{ Mother's BMI (kg/m2) } \\
\hline Underweight $(<18.5)$ & 38 & 1.4 & 3.3 & 46 & 1.8 & 5.3 & 55 & 2.1 & 3.5 & 140 & 1.8 \\
\hline Normal (18.5-24.9) & 1130 & 42.5 & 8.4 & 1051 & 39.9 & 10.6 & 963 & 36.6 & 10.4 & 3144 & 39.7 \\
\hline Overweight (25-29.9) & 923 & 34.7 & 10.2 & 923 & 35.0 & 12.1 & 959 & 36.4 & 10.9 & 2805 & 35.4 \\
\hline Obese $(\geq 30)$ & 569 & 21.4 & 13.2 & 614 & 23.3 & 10.7 & 655 & 24.9 & 15.0 & 1838 & 23.2 \\
\hline \multicolumn{12}{|l|}{ Mother's mother tongue } \\
\hline Turkish & 1937 & 71.8 & 10.6 & 1994 & 74.3 & 11.2 & 1888 & 70.3 & 12.5 & 5820 & 72.1 \\
\hline Kurdish & 657 & 24.3 & 8.4 & 596 & 22.2 & 10.3 & 676 & 25.2 & 9.9 & 1929 & 23.9 \\
\hline Other & 104 & 3.9 & 9.9 & 94 & 3.5 & 10.7 & 122 & 4.5 & 7.4 & 320 & 4.0 \\
\hline \multicolumn{12}{|l|}{ Mother's education } \\
\hline No/incomplete primary school & 676 & 25.1 & 9.9 & 539 & 20.1 & 10.0 & 532 & 19.8 & 6.9 & 1747 & 21.7 \\
\hline Primary school & 1418 & 52.5 & 10.7 & 1366 & 50.9 & 10.4 & 965 & 35.9 & 11.7 & 3748 & 46.5 \\
\hline Secondary school & 213 & 7.9 & 5.5 & 250 & 9.3 & 12.4 & 493 & 18.3 & 14.7 & 956 & 11.8 \\
\hline High school and higher & 391 & 14.5 & 10.2 & 529 & 19.7 & 12.8 & 697 & 25.9 & 13.0 & 1618 & 20.1 \\
\hline
\end{tabular}


Table 1. (Continued)

\begin{tabular}{|c|c|c|c|c|c|c|c|c|c|c|c|}
\hline & \multicolumn{3}{|c|}{ TDHS-2003 } & \multicolumn{3}{|c|}{ TDHS-2008 } & \multicolumn{3}{|c|}{ TDHS-2013 } & \multicolumn{2}{|c|}{ Total } \\
\hline & $n$ & $\%$ & $\% O w^{*}$ & $n$ & $\%$ & $\% O w^{*}$ & $n$ & $\%$ & $\% O w^{*}$ & $n$ & $\%$ \\
\hline \multicolumn{12}{|l|}{ Mother's employment status } \\
\hline Not employed & 1759 & 65.2 & 9.9 & 2117 & 78.9 & 10.5 & 2170 & 80.8 & 12.4 & 6046 & 74.9 \\
\hline Employed without social security & 805 & 29.9 & 9.9 & 393 & 14.6 & 12.8 & 293 & 10.9 & 5.2 & 1491 & 18.5 \\
\hline Employed with social security & 133 & 4.9 & 12.0 & 174 & 6.5 & 12.9 & 224 & 8.3 & 12.9 & 531 & 6.6 \\
\hline \multicolumn{12}{|l|}{ Father's education } \\
\hline No/incomplete primary school & 235 & 8.7 & 10.7 & 143 & 5.3 & 6.7 & 199 & 7.4 & 10.5 & 576 & 7.1 \\
\hline Primary school & 1386 & 51.4 & 9.6 & 1290 & 48.1 & 11.0 & 1003 & 37.3 & 8.8 & 3679 & 45.6 \\
\hline Secondary school & 325 & 12.0 & 11.5 & 395 & 14.7 & 10.0 & 512 & 19.0 & 13.0 & 1231 & 15.3 \\
\hline High school and higher & 752 & 27.9 & 9.9 & 856 & 31.9 & 12.2 & 973 & 36.2 & 14.1 & 2581 & 32.0 \\
\hline \multicolumn{12}{|l|}{$\begin{array}{l}\text { Individual-level (child) } \\
\text { characteristics }\end{array}$} \\
\hline \multicolumn{12}{|l|}{ Age of child (months) } \\
\hline $0-11$ & 515 & 19.4 & 10.8 & 495 & 18.4 & 9.4 & 521 & 19.4 & 9.6 & 1530 & 19.1 \\
\hline $12-23$ & 490 & 18.5 & 13.6 & 578 & 21.6 & 16.9 & 554 & 20.6 & 18.1 & 1622 & 20.2 \\
\hline $24-35$ & 542 & 20.4 & 11.3 & 535 & 19.9 & 14.7 & 498 & 18.5 & 14.3 & 1575 & 19.6 \\
\hline $36-47$ & 535 & 20.2 & 10.2 & 515 & 19.2 & 6.7 & 550 & 20.5 & 7.2 & 1600 & 21.0 \\
\hline $48-59$ & 572 & 21.6 & 4.1 & 560 & 20.9 & 6.8 & 564 & 21.0 & 9.1 & 1696 & 21.1 \\
\hline \multicolumn{12}{|l|}{ Sex of child } \\
\hline Female & 1407 & 52.2 & 11.6 & 1406 & 52.4 & 12.2 & 1451 & 54.0 & 12.2 & 4264 & 52.9 \\
\hline Male & 1290 & 47.8 & 8.4 & 1278 & 47.6 & 9.7 & 1235 & 46.0 & 10.9 & 3804 & 47.1 \\
\hline \multicolumn{12}{|l|}{ Type of birth } \\
\hline Normal & 2110 & 78.3 & 10.0 & 1658 & 61.8 & 10.6 & 1358 & 50.7 & 11.0 & 5126 & 63.6 \\
\hline Caesarean & 586 & 21.7 & 10.0 & 1026 & 38.2 & 11.6 & 1320 & 49.3 & 12.3 & 2932 & 36.4 \\
\hline \multicolumn{12}{|l|}{ Duration of breast-feeding } \\
\hline$<6$ months & 649 & 24.2 & 16.9 & 426 & 15.9 & 11.7 & 332 & 15.3 & 10.8 & 1408 & 18.7 \\
\hline$\geq 6$ months & 1795 & 66.8 & 8.7 & 2055 & 76.8 & 11.3 & 1638 & 75.5 & 13.3 & 5488 & 72.8 \\
\hline $\begin{array}{l}\text { The child is under than } 6 \\
\text { months old and is still } \\
\text { breastfeeding }\end{array}$ & 243 & 9.0 & 12.7 & 194 & 7.3 & 7.3 & 200 & 9.2 & 7.1 & 637 & 8.5 \\
\hline \multicolumn{12}{|l|}{ Bottle-feeding } \\
\hline No & 1704 & 64.3 & 8.5 & 1594 & 59.5 & 9.3 & 1586 & 59.2 & 11.0 & 4884 & 61.0 \\
\hline Yes & 946 & 35.7 & 12.4 & 1086 & 40.5 & 13.5 & 1095 & 40.8 & 12.7 & 3127 & 39.0 \\
\hline \multicolumn{12}{|l|}{ Birth weight } \\
\hline Low (<2500 g) & 201 & 10.4 & 12.6 & 224 & 9.7 & 7.2 & 254 & 9.9 & 12.7 & 679 & 9.9 \\
\hline Middle (2500-4000 g) & 1500 & 77.2 & 9.0 & 1830 & 79.0 & 11.7 & 2106 & 81.9 & 11.4 & 5435 & 79.6 \\
\hline High (>4000 g) & 241 & 12.4 & 14.4 & 262 & 11.3 & 13.5 & 210 & 8.2 & 17.6 & 714 & 10.5 \\
\hline \multicolumn{12}{|l|}{ Stunted } \\
\hline No & 2307 & 85.5 & 9.4 & 2363 & 88.0 & 10.6 & 2428 & 90.4 & 10.6 & 7097 & 88.0 \\
\hline Yes & 391 & 14.5 & 13.9 & 321 & 12.0 & 13.8 & 259 & 9.6 & 21.0 & 971 & 12.0 \\
\hline
\end{tabular}


Table 2. Relationship between overweight of children under five years in Turkey and household/maternal and children characteristics'; pooled data from Turkey Demographic and Health Survey 2003, 2008 and 2013 (Weighted)

\begin{tabular}{|c|c|c|c|c|}
\hline & \multirow[b]{2}{*}{$\mathrm{n}$} & \multicolumn{2}{|c|}{ Overweight/Obese } & \multirow[b]{2}{*}{$p$-value } \\
\hline & & Yes $n(\%)$ & No $n(\%)$ & \\
\hline \multicolumn{5}{|l|}{ Household/maternal characteristics } \\
\hline Place of residence & 8068 & & & 0.32 \\
\hline Rural & & $226(10.3)$ & $1963(89.7)$ & \\
\hline Urban & & $653(11.1)$ & $5226(88.9)$ & \\
\hline Wealth index quintile & 8069 & & & 0.01 \\
\hline Lowest (poorest) & & $181(9.7)$ & $1685(90.3)$ & \\
\hline Second & & $189(10.3)$ & $1650(89.7)$ & \\
\hline Middle & & $168(10.1)$ & $1494(89.9)$ & \\
\hline Fourth & & $181(12.3)$ & $1288(87.7)$ & \\
\hline Highest (richest) & & $160(13.0)$ & $1073(87.0)$ & \\
\hline Marital status & 8068 & & & 0.17 \\
\hline Couple & & $865(10.8)$ & $7112(89.2)$ & \\
\hline Single parent & & $14(15.4)$ & $77(84.6)$ & \\
\hline Mother's age (years) & 8067 & & & 0.65 \\
\hline $15-19$ & & $22(11.2)$ & $174(88.8)$ & \\
\hline $20-29$ & & $486(11.2)$ & $3868(88.8)$ & \\
\hline$\geq 30$ & & $370(10.5)$ & $3147(89.5)$ & \\
\hline Mother's BMI $\left(\mathrm{kg} / \mathrm{m}^{2}\right)$ & 7925 & & & $<0.001$ \\
\hline Underweight $(<18.5)$ & & $6(4.3)$ & $134(95.7)$ & \\
\hline Normal (18.5-24.9) & & $307(9.8)$ & $2837(90.2)$ & \\
\hline Overweight (25-29.9) & & $310(11.1)$ & $2494(88.9)$ & \\
\hline Obese $(\geq 30)$ & & $239(13.0)$ & $1598(87.0)$ & \\
\hline Mother's mother tongue & 8067 & & & 0.04 \\
\hline Other & & $29(9.1)$ & $290(90.9)$ & \\
\hline Kurdish & & $184(9.5)$ & $1745(90.5)$ & \\
\hline Turkish & & $665(11.4)$ & $5154(88.6)$ & \\
\hline Mother's education & 8068 & & & 0.01 \\
\hline No/incomplete primary school & & $157(9.0)$ & $1590(91.0)$ & \\
\hline Primary school & & $408(10.9)$ & $3340(89.1)$ & \\
\hline Secondary school & & $115(12.0)$ & $840(88.0)$ & \\
\hline High school and higher & & $199(12.3)$ & $1419(87.7)$ & \\
\hline Mother's employment status & 8068 & & & 0.17 \\
\hline Not employed & & $666(11.0)$ & $5380(89.0)$ & \\
\hline Employed without social security & & $146(9.8)$ & $1345(90.2)$ & \\
\hline Employed with social security & & $67(12.6)$ & $464(87.4)$ & \\
\hline
\end{tabular}


Table 2. (Continued)

\begin{tabular}{|c|c|c|c|c|}
\hline & \multirow[b]{2}{*}{$\mathrm{n}$} & \multicolumn{2}{|c|}{ Overweight/Obese } & \multirow[b]{2}{*}{$p$-value } \\
\hline & & Yes $n(\%)$ & No $n(\%)$ & \\
\hline Father's education & 8068 & & & 0.014 \\
\hline No/incomplete primary school & & $56(9.7)$ & $521(90.3)$ & \\
\hline Primary school & & $363(9.9)$ & $3315(90.1)$ & \\
\hline Secondary school & & $143(11.6)$ & $1088(88.4)$ & \\
\hline High school and higher & & $317(12.3)$ & $2265(87.7)$ & \\
\hline \multicolumn{5}{|l|}{ Individual-level (child) characteristics } \\
\hline Age of child (months) & 8025 & & & $<0.001$ \\
\hline $0-12$ & & $152(9.9)$ & $1378(90.1)$ & \\
\hline $13-24$ & & $265(16.3)$ & $1358(83.7)$ & \\
\hline $25-36$ & & $211(13.4)$ & $1364(86.6)$ & \\
\hline $37-59$ & & $242(7.3)$ & $3055(92.7)$ & \\
\hline Sex of child & 8068 & & & 0.001 \\
\hline Female & & $367(9.6)$ & $3437(90.4)$ & \\
\hline Male & & $512(12.0)$ & $3752(88.0)$ & \\
\hline Type of birth & 8057 & & & 0.13 \\
\hline Normal & & $538(10.5)$ & $4588(89.5)$ & \\
\hline Caesarean & & $340(11.6)$ & $2591(88.4)$ & \\
\hline Duration of breast-feeding & 7532 & & & 0.31 \\
\hline The child is under 6 months old and is still breastfeeding & & $59(9.3)$ & $578(90.7)$ & \\
\hline$<6$ months & & $162(11.5)$ & $1246(88.5)$ & \\
\hline$\geq 6$ months & & $606(11.0)$ & $4881(89.0)$ & \\
\hline Bottle-feeding & 8011 & & & $<0.001$ \\
\hline No & & $467(9.6)$ & $4417(90.4)$ & \\
\hline Yes & & $402(12.9)$ & $2725(87.1)$ & \\
\hline Birth weight & 6828 & & & 0.004 \\
\hline Low $(<2500 \mathrm{~g})$ & & $74(10.9)$ & $605(89.1)$ & \\
\hline Middle $(2500-4000 \mathrm{~g})$ & & $588(10.8)$ & $4847(89.2)$ & \\
\hline High (>4000 g) & & $107(15.0)$ & $607(85.0)$ & \\
\hline Stunted & 8069 & & & $<0.001$ \\
\hline No & & $726(10.2)$ & $6372(89.8)$ & \\
\hline Yes & & $153(15.8)$ & $818(84.2)$ & \\
\hline Birth cohort & 8069 & & & 0.17 \\
\hline $1999-2003$ & & $271(10.0)$ & $2427(90.0)$ & \\
\hline $2004-2008$ & & $295(11.0)$ & $2389(89.0)$ & \\
\hline $2009-2013$ & & $313(11.6)$ & $2374(88.4)$ & \\
\hline
\end{tabular}


Table 3. Summary measures of between-cluster effects on overweight and obesity in children under five years

\begin{tabular}{|c|c|c|c|c|}
\hline & Null model & Model 2 & Model 3 & Model 4 \\
\hline Variance (SD) & $0.597(0.773)$ & $0.539(0.734)$ & $0.620(0.787)$ & $0.582(0.763)$ \\
\hline ICC, \% & 15.36 & 14.07 & 15.85 & 15.05 \\
\hline PCV, \% & ref & 9.72 & -3.85 & 2.51 \\
\hline MOR & 2.09 & 2.01 & 2.12 & 2.07 \\
\hline$-2 L L$ & 4866.7 & 4823.9 & 4694.9 & 4646.9 \\
\hline
\end{tabular}

variance: cluster-level variance, SD: standard deviation, ICC: intra-class correlation coefficient, PCV: Proportional change in variance, MOR: Median odds ratio, -2LL: - $2 \times \log$-likelihood; null model (model 1 ) is random intercept the only model with no predictors, model 2 included household/maternal variables, model 3 included individual-level variables, model 4 is a final model with both household/maternal and individual-level variables

Model 2 was a multilevel model adjusted for maternal/household level characteristics. The probability of having an overweight/obese child was higher among all the three maternal BMI groups compared to underweight women. Maternal BMI was associated with childhood overweight/obesity - having an obese mother $(\mathrm{aOR}=3.70,95 \% \mathrm{CI}=1.51-9.09)$, overweight mother $(\mathrm{aOR}=2.84,95 \% \mathrm{CI}=1.16-6.96)$ and normal-BMI mother $(\mathrm{aOR}=2.57,95 \% \mathrm{CI}=1.05-6.28)$ compared to having an underweight mother.

In Model 3 - comprising the individual-level characteristics - one-year-old children were more prone to be overweight/obese $-72 \%(95 \% \mathrm{CI}=1.30-2.28)$, compared to zero-year-old children. Also, children over 36 months old had lower odds ( $\mathrm{aOR}=0.63,95 \% \mathrm{CI}=0.47-0.84$ ) of being overweight. When examined by sex, overweight/obesity in male children was 1.32 (95\% $\mathrm{CI}=1.13-1.55)$ times higher than in females. Children having high birth weight were 1.72 $(95 \% \mathrm{CI}=1.20-2.47)$ times more likely to be overweight/obese compared to children having low birth weight. Overweight/obesity in stunted children was $2.05(95 \% \mathrm{CI}=1.64-2.55)$ times higher than children who weren't stunted.

Model 4 included both maternal/household and individual-level characteristics. Overweight/obesity was $2.27(95 \% \mathrm{CI}=1.21-4.26)$ times more common among children living in single-parent households compared to those living in dual-parent households. As the mother's BMI increased, the frequency of overweight/obesity increased in children. Children having normal-BMI mothers were 2.70 (95\% CI =1.11-6.59), having overweight mothers were 3.15 (95\% CI = 1.29-7.69), and having obese mothers were $4.25(95 \% \mathrm{CI}=1.73-10.44)$ times more likely to be overweight/ obese compared to children having an underweight mother. Overweight was higher among children of mothers who have completed primary school ( $\mathrm{aOR}=1.21,95 \% \mathrm{CI}=1.01$ to 1.59 ) than children of mothers who have not completed primary school. One-year-old children had a $75 \%$ higher risk (95\% CI $=1.34-2.28)$ of being overweight/obese than children aged 0-12 months. Conversely, children over the age of three had a $40 \%$ lower probability of being overweight/obese than children aged 0-12 months. In addition, overweight/obesity in male children was $1.30(95 \% \mathrm{CI}=1.11-1.53)$ times higher than females. Children with high birth weight had a 55\% (95\% CI $=1.08-2.23)$ higher probability of being overweight/obese compared to children with low birth weight. Stunting in preschool children was a constituent that accompanied overweight/obesity. Stunted children were 2.18 (95\% $\mathrm{CI}=1.74-2.73)$ times more overweight/obese compared to children who weren't stunted.

\section{Discussion}

This study examined the relationship between selected socio-economic, bio-demographic variables and the overweight status of under-five-year-old children in three consecutive TDHSs between 2003 and 2013; and identified the 25-year prevalence of overweight/obesity using TDHS 1993, 1998, 2003, 2008, and 2013 datasets. There is extensive literature in examining 
Table 4. A multilevel analysis of overweight in children under five years by household/maternal and individual-level characteristics; pooled data from Turkey Demographic and Health Survey 2003, 2008, and 2013 (Weighted)

\begin{tabular}{|c|c|c|c|}
\hline & $\begin{array}{c}\text { Model } 2 \\
\text { aOR }(95 \% \mathrm{Cl})\end{array}$ & $\begin{array}{c}\text { Model } 3 \\
\text { aOR (95\% Cl) }\end{array}$ & $\begin{array}{c}\text { Model } 4 \\
\text { aOR }(95 \% \mathrm{Cl})\end{array}$ \\
\hline \multicolumn{4}{|l|}{ Household/maternal characteristics } \\
\hline \multicolumn{4}{|l|}{ Household residence } \\
\hline Rural & ref & & ref \\
\hline Urban & $0.85(0.68-1.07)$ & & $0.88(0.70-1.11)$ \\
\hline \multicolumn{4}{|l|}{ Wealth index quintile } \\
\hline Lowest (poorest) & ref & & ref \\
\hline Second & $1.05(0.81-1.36)$ & & $1.07(0.82-1.40)$ \\
\hline Middle & $0.99(0.74-1.31)$ & & $1.01(0.75-1.36)$ \\
\hline Fourth & $1.13(0.83-1.53)$ & & $1.23(0.90-1.69)$ \\
\hline Highest (richest) & $1.26(0.88-1.79)$ & & $1.37(0.95-1.97)$ \\
\hline \multicolumn{4}{|l|}{ Marital status } \\
\hline Couple & ref & & ref \\
\hline Single parent (mother) & $1.81(0.98-3.33)$ & & $2.27(1.21-4.26)^{\star}$ \\
\hline \multicolumn{4}{|l|}{ Mother's age (years) } \\
\hline $15-19$ & ref & & ref \\
\hline $20-29$ & $0.79(0.48-1.28)$ & & $0.85(0.51-1.40)$ \\
\hline$\geq 30$ & $0.67(0.41-1.11)$ & & $0.77(0.46-1.30)$ \\
\hline \multicolumn{4}{|l|}{ Mother's BMI $\left(\mathrm{kg} / \mathrm{m}^{2}\right)$} \\
\hline Underweight $(<18.5)$ & ref & & ref \\
\hline Normal (18.5-24.9) & $2.57(1.05-6.28)^{\star}$ & & $2.70(1.11-6.59)^{\star}$ \\
\hline Overweight (25-29.9) & $2.84(1.16-6.96)^{*}$ & & $3.15(1.29-7.69)^{\star}$ \\
\hline Obese $(\geq 30)$ & $3.70(1.51-9.09)^{\star}$ & & $4.25(1.73-10.44)^{*}$ \\
\hline \multicolumn{4}{|l|}{ Mother's mother tongue } \\
\hline Other & ref & & ref \\
\hline Kurdish & $1.07(0.66-1.73)$ & & $1.11(0.67-1.81)$ \\
\hline Turkish & $1.01(0.64-1.60)$ & & $1.12(0.70-1.80)$ \\
\hline \multicolumn{4}{|l|}{ Mother's education } \\
\hline No/incomplete primary school & ref & & ref \\
\hline Primary school & $1.31(1.03-1.70)^{*}$ & & $1.21(1.01-1.59)$ \\
\hline Secondary school & $1.33(0.94-1.87)$ & & $1.18(0.83-1.67)$ \\
\hline High school and higher & $1.29(0.91-1.84)$ & & $1.12(0.78-1.61)$ \\
\hline \multicolumn{4}{|l|}{ Mother's employment status } \\
\hline Not employed & ref & & ref \\
\hline Employed without social security & $0.93(0.74-1.17)$ & & $0.92(0.73-1.16)$ \\
\hline Employed with social security & $1.06(0.76-1.48)$ & & $1.15(0.81-1.63)$ \\
\hline
\end{tabular}


Table 4. (Continued)

\begin{tabular}{|c|c|c|c|}
\hline & $\begin{array}{c}\text { Model } 2 \\
\text { aOR }(95 \% \mathrm{Cl})\end{array}$ & $\begin{array}{c}\text { Model } 3 \\
\text { aOR }(95 \% \mathrm{Cl})\end{array}$ & $\begin{array}{c}\text { Model } 4 \\
\text { aOR }(95 \% \mathrm{Cl})\end{array}$ \\
\hline \multicolumn{4}{|l|}{ Father's education } \\
\hline No/incomplete primary school & ref & & Ref \\
\hline Primary school & $0.98(0.68-1.40)$ & & $0.94(0.66-1.35)$ \\
\hline Secondary school & $1.07(0.72-1.60)$ & & $1.02(0.68-1.53)$ \\
\hline High school and higher & $1.13(0.76-1.68)$ & & $1.10(0.74-1.64)$ \\
\hline \multicolumn{4}{|l|}{ Individual-level (child) characteristics } \\
\hline \multicolumn{4}{|l|}{ Age of child (months) } \\
\hline $0-12$ & & ref & ref \\
\hline $13-24$ & & $1.72(1.30-2.28)^{\star}$ & $1.75(1.34-2.28)^{*}$ \\
\hline $25-36$ & & $1.27(0.95-1.70)$ & $1.27(0.95-1.70)$ \\
\hline $37-59$ & & $0.63(0.47-0.84)^{\star}$ & $0.60(0.45-0.81)^{*}$ \\
\hline \multicolumn{4}{|l|}{ Sex of child } \\
\hline Female & & ref & ref \\
\hline Male & & $1.32(1.13-1.55)^{\star}$ & $1.30(1.11-1.53)^{\star}$ \\
\hline \multicolumn{4}{|l|}{ Type of birth } \\
\hline Normal & & ref & ref \\
\hline Caesarean & & $0.99(0.83-1.18)$ & $0.91(0.76-1.09)$ \\
\hline \multicolumn{4}{|l|}{ Duration of breast-feeding } \\
\hline $\begin{array}{l}\text { The child is under } 6 \text { months old and is still breast- } \\
\text { feeding }\end{array}$ & & ref & ref \\
\hline$<6$ months & & $1.23(0.81-1.86)$ & $1.19(0.79-1.81)$ \\
\hline$\geq 6$ months & & $1.13(0.77-1.66)$ & $1.13(0.77-1.66)$ \\
\hline \multicolumn{4}{|l|}{ Bottle-feeding } \\
\hline No & & ref & ref \\
\hline Yes & & $1.12(0.94-1.33)$ & $1.10(0.93-1.31)$ \\
\hline \multicolumn{4}{|l|}{ Birth weight } \\
\hline Low $(<2500 \mathrm{~g})$ & & ref & ref \\
\hline Middle $(2500-4000 \mathrm{~g})$ & & $1.15(0.86-1.55)$ & $1.08(0.80-1.44)$ \\
\hline High $(>4000 \mathrm{~g})$ & & $1.72(1.20-2.47)^{\star}$ & $1.55(1.08-2.23)^{*}$ \\
\hline \multicolumn{4}{|l|}{ Stunted } \\
\hline No & & ref & ref \\
\hline Yes & & $2.05(1.64-2.55)^{\star}$ & $2.18(1.74-2.73)^{\star}$ \\
\hline
\end{tabular}

${ }^{\star} \mathrm{p}<0.05$

the factors of child nutritional status in a wide range of countries, utilizing different analytical methods. However, previous studies have mostly used standard multiple linear or logistic regressions. DHS data have a hierarchical structure, with children nested within women, women clustered within households, and households nested within communities. Observations from the same 
group are expected to be more alike at least in part because they share the common characteristics or have been exposed to a habitual set of conditions, thus violating the standard assumption of independence of observations native in conventional regression models. As a result, in terms of analysing such data, standard statistical methods are expected to lead to valid results unless some allowance for clustering is made (Duncan et al., 1998; Rasbash et al., 2013). Multilevel models are technically stronger as well as provide an analytical structure with a much greater capacity for generality when compared to the conventional single-level statistical methods (Raudenbush \& Bryk, 2002). For example, in a recent study using logistic regression in the TDHS-2013 data set, Santas and Santas (2018) found that, contrary to the findings in this study, overweight/obesity was found to be significantly more common among female children than males. In two studies using similar anthropometric criteria in the same data set, the reason for finding different overweight/obesity-related factors could be due to the use of statistically different methods. Therefore, taking the clustered data structure into account, in this study, multilevel logistic regression models are fitted to assess the relationship between bio-demographic, socioeconomic variables, and overweight status.

Childhood obesity is associated with critical health problems and the risk of early NCDs. Studies have highlighted that the body weight of children under five years is a substantial predictor of health at older ages (Brophy et al., 2009; Deckelbaum \& Williams, 2001). Monitoring related trends are important for combating obesity in early childhood. The findings of this study show that the prevalence of overweight/obesity in preschool children in Turkey was 5.3\% in 1993; $4.9 \%$ in $1998 ; 10.0 \%$ in $2003 ; 11 \%$ in 2008 ; and $11.6 \%$ in 2013 . Preschool children's overweight/obesity in Turkey were presented for the first time in 2013 TDHS. In this report, children who were two standard deviations above the median weight-for-height were considered overweight/obese and $11 \%$ of children under the age of five were found to be overweight/obese. The WHO recommends assessing obesity in preschool children with the BMI-for-age which is also the globally preferred measurement (WHO, 2008). This study identified overweight/obesity based on BMI-for-age instead of weight-for-length/height and weight-for-age. Nevertheless, weight-for-length/height and BMI-for-age yield close results, which suggests comparability between indicators for assessing overweight/obesity in children aged under five (De Onis et al., 2010). This study has already shown that the 2013 results were similar to the weight-for-height measurement in the TDHS2013 report ( $11.6 \%$ vs $10.9 \%)$.

On a large scale, Turkey Nutrition and Health Survey (TNHS) conducted in 2010, which included 2567 preschool children, found the rate of overweight/obesity (BMI-for-age Z-score $\geq+2 \mathrm{SD}$ ) as $8.5 \%$. In TNHS, the researcher or the mother might have chosen the healthier child and therefore the actual prevalence might have been higher $(\mathrm{MOH} / \mathrm{HUBDB} / \mathrm{NH}, 2010)$. The results of this study are consistent with the results of other studies in Turkey. For example, in a 2003 study conducted in Izmir (Ucar et al., 2009) - one of the larger cities in Turkey - found the prevalence of overweight among children aged 2-6 years as $10.3 \%$. Also, in a cross-sectional study (Kondolot et al., 2011) conducted in Kayseri, Turkey, in 2010, the prevalence of overweight among children aged 0-84 months was found as $10 \%$.

In another study of Santas and Santas (2018), which was an advanced analysis performed with TDHS-2013 data, the frequency of overweight/obesity under the age of five, based on weight-forheight indicator, was found to be $8.6 \%$. Z scores based on both the NCHS reference and the recent WHO reference are presented in the 2013-TDHS data set. When the data set in the study of Santas and Santas were examined, it was determined that obesity was evaluated according to the NHCS references, which was used before 2006. In this study, Z scores for TDHS-1993, TDHS-1998, and TDHS-2003 were revised in accordance with 2006 WHO Child Growth Standards, which is the current reference system instead of NCHS references. For the TDHS-2008 and TDHS-2013, $\mathrm{Z}$ scores that were based on the recent WHO reference was used. Thus, overweight levels that are comparable globally and between TDHS series were obtained. WHO reports that the current 
obesity epidemic could be detected earlier in many developed countries if new international standards were applied 20 years ago (WHO, 2019b).

In this study, a doubled prevalence of overweight/obesity among preschool children was found in the early 2000s. The prevalence, which was around 5\% before 2000 , increased to $10 \%$ in 2003 . De Onis and Blössner (2000) reported on the prevalence of overweight among preschool children based on data from 94 countries from 160 nationally representative samples. The worldwide prevalence of overweight was $3.3 \%$, and it was found to have increased in 16 of the 38 countries. In 2010, another study by De Onis et al., reported that the global prevalence of preschool children overweight/obesity increased from $4.2 \%$ in 1990 to $6.7 \%$ in 2010 , for a relative increase of $60 \%$. The observed increase in the prevalence of preschool childhood overweight/obesity after 2000 is a likely result of a change in nutrition and physical activity patterns over time. Modernization, urbanization, and the occurrence of contemporary problems such as screen addiction as a result of technological development have led to a decrease in physical activity. This behavioural change is influenced by plural environmental and social factors, including community, national policies, interpersonal, and interaction with biological processes (Huang and Glass, 2008).

The prevalence of overweight is lowest in Central East Anatolia (5.9\%) and highest in Western regions (\%13.8 and 13.4\%) and Istanbul (13.2\%) where urbanization is high. In the TDHS reports, the Eastern region has been defined as the least developed region of the country (HIPS, 2014). This finding indicates the inequality between regions, and the need for regional priority for intervention planning.

In the present study, maternal BMI showed strong associations with childhood overweight/ obesity, even after controlled for all other socioeconomic and demographic variables. The findings of this study indicate the child of an obese mother is at a greater risk - 4.5 times more - for being overweight than an underweight mother, and this increase in risk correlates positively with the increase in the mother's BMI. The observed relationship is similar to the results from other studies (Danielzik et al., 2004; Portela et al., 2015). Armoon and Karimy's (2019) also showed that, in Iran, the mother's obesity increased the risk of the child's obesity by 4 times. In a similar survey on 3 to 5 -year-old children, mother's obesity was one of the important associated factors of childhood obesity (Gewa, 2010). Familial patterns of obesity result from genes, environment, and behavioural factors working in harmony, especially for young children growing up within the family. It is unlikely that these factors can be considered to work independently. That is, family members with a genetic tendency for obesity are likely to share similar environments, including high fat diets and low levels of activity, that lead to overweight (Birch and Davison, 2001). Some studies have shown high dietary fat intake to be a significant predictor of obesity among women only when there was familial propensity (Pérusse and Bouchard, 2000). Due to mothers and children exposed to an obesogenic environment that may predispose them to increased risks for the development of overweight, children whose parents consumed high levels of total fat, saturated fat, and cholesterol have been shown to consume high levels of these nutrients (Oliveria et al., 1992). Overweight mothers are also inclined to not practice in breastfeeding, which leads to their children not benefiting from any preventive impact that breastfeeding may provide (Hediger et al., 2001). Research studies have also shown that children born to obese mothers are at an increased risk of macrosomia and congenital abnormalities, as well as being obese later in life (Leddy et al., 2008; Sorrie et al., 2017).

The results of this study indicate that the overweight rate is higher among children who live in a single parent household. These findings are similar to previous conclusions of some studies (Duriancik and Goff, 2019). Schmeer (2012) examined the impacts of family structure on obesity among children of age from 3 to 5 . Schmeer found that single parenthood resulted in higher BMI scores among the children compared to those children in dual-parent households. Chen and Escarce (2010) also concluded that children in single-parent households were at the greatest risk for childhood obesity. Possible explanations for this relationship are that children in single-parent households typically consume foods higher in saturated fat and sugar; have less access to 
vegetables and fruits; watch more TV (Byrne et al., 2011; Huffman et al., 2010); and do less physical activity (Augustine and Kimbro, 2017; Duriancik and Goff, 2019) than children from twoparent households. Also, financial constraints, greater instability, and unshared responsibilities in housework in single-parent families may be other potential challenges (Duriancik and Goff, 2019; Sisson et al., 2014; Yelick, 2017). A current study (Irvin et al., 2018) illustrated that children in single-parent houses had a higher percentage of unmet health-care needs compared to children in dual-parent houses.

This study also implied that the maternal education level and the children's overweight status are related. Overweight is higher among children of mothers who have completed primary school than children of uneducated mothers. It seems that there is a trend in this relationship when the bivariate analysis is considered; as the mother's education level increases, the percentage of children that are overweight increases (Table 2). Even though no significant evidence was found in multilevel regression analysis, a similar relationship was also discovered for the education level of the father, in the bivariate analysis. As the father's education level increases, the percentage of children that are overweight increases. On the other hand, research on the association between maternal education level and overweight/obesity in early childhood did not lead to a definite conclusion. Recent studies across 11 European countries have shown that low maternal education could yield a significant risk of early childhood obesity (Ruiz et al., 2016). Interestingly, the relationship between maternal education and children's overweight status in China and Kenya is different from that in western countries. In the case of China and Kenya, the overweight rate among children with a high maternal education level is higher than that of lower maternal education level (Gewa, 2010; Liu et al., 2016). The possible explanation could be that in the given social conditions, mothers who received higher education could find a better position of employment and command higher incomes. Evaluating this relationship with wealth status may yield more accurate results. Also, it should be noted that, while in high-income countries, children living in low socioeconomic level households have higher obesity rates, in developing countries children living in higher socioeconomic levels have higher obesity rates (Han et al., 2010; McCormick et al., 2010).

Another important finding in this study the relationship between age and overweight among children. This study showed an increase in the likelihood of being overweight at 13-23 months of age than children aged 0-12 months. Previous studies reported that overweight was more common in infants (McCormick et al., 2010; Tchoubi et al., 2015). TBSA-2010, a national study conducted in Turkey, the prevalence of overweight under 5 years of age was the highest in the 19-24-month age group (15\%) (MOH/HUBDB/NH, 2010). In addition, the present study showed that children over 36 months old had significantly lower odds of being overweight. After the age of 3, there may be a relationship due to the increase in physical activity, with the development of children's motor skills to a great extent and the ability to go out and play games.

The analyses showed that males had a greater chance of being overweight compared to females. The link between sex and overweight in children is inconsistent across the literature but male predominance was observed in many studies (Benedict et al., 2020; Chen et al., 2016; El-Gamal et al., 2020; Kurspahić-Mujčić and Mujčić, 2020; Tchoubi et al., 2015). However, in some studies female predominance (Jouret et al., 2007), or no difference was observed (Gewa, 2010; Ogden et al., 2012; Reilly et al., 2005), supporting the theory that overweight was an outcome of interactions between genetics, behaviour, and environmental factors.

Birth weight is a strong factor related to the occurrence of overweight/obesity among preschool children studied. Not surprisingly, birth weight higher than 4000 grams involved a high risk for the occurrence of overweight in preschool years. These findings are consistent with previous reports (Armoon and Karimy, 2019; Gewa, 2010; Oppitz et al., 2014; Tchoubi et al., 2015; Yu et al., 2011). The relation between high birth weight and the increased risk of childhood overweight/obesity was attributed to endocrine and metabolic pathways. Moreover, the high birth weight indicated the danger of obesity not only at the preschool-ages but also during the rest of the childhood (Qiao et al., 2015; Rugholm et al., 2005; Yu et al., 2011). The report of a 
meta-analysis-based study indicates with high consistency that the high birth weight may lead to a doubling of the long-term overweight risk, regardless of sex, socio-economic status, geographic/ ethnic origin, parental weight status, etc. Accordingly, prenatal overfeeding is a key risk factor leading to long-term obesity predisposition (Schellong et al., 2012).

The results of the multilevel regression showed that, in stunted children, the chance of becoming overweight was more than 2 times higher than non-stunted children. This phenomenon has been found consistently in many countries (El-Gamal et al., 2020; Gewa, 2010; Mamabolo et al., 2005; Said-Mohamed et al., 2012; Steyn et al., 2005; Tzioumis and Adair, 2014; Wang et al., 2009). Similar circumstances, whether behavioural or environmental factors favour the growth of stunting and overweight in the same household. The mechanism of the association between stunting and overweight is still not conclusive. It has been assumed that nutritional stunting is related to impaired fat oxidation (Hoffman et al., 2000b). Secondly, another theory is that stunted children may have a damaged regulation of energy intake, which results in the risk of being overweight (Hoffman et al., 2000a). Thirdly, mild stunting might be related to a greater sensitivity to the effects of high-fat diets (Sawaya et al., 1998). Furthermore, low physical activity is a substantial risk factor for stunting and being overweight (Said-Mohamed et al., 2012). The double burden should be noteworthy in preschool children in Turkey because the focus of health services should also be overweight as well as undernutrition.

This study implies that, as the household's wealth index increases, the percentage of obesity in children increases slightly (Table 2). Although this relationship was statistically significant in bivariate analysis, it was not significant in multilevel analysis. Wealth status has been related to health outcomes such as nutritional status, as it is directly linked to the accessibility of nutrition-rich food and living in a healthful environment. This difference may be related to the fact that poor households in high-income countries have easy access to carbohydrate and fat-rich, non-nutritious, obesogenic foods, and in developing countries, the increase in purchasing power and the shift to richer households in access to obesogenic foods.

Although BMI is the main indicator of obesity, various methods are available for the determination of body fat content, such as bioelectrical impedance analysis (BIA), skinfold thickness (SFT), isotope dilution method, measurement of dual energy X-ray absorption (DEXA), and measurement of body K. However, measurement of BMI alone may have limitations in some populations and ethnicities, as it does not distinguish between increases in body weight attributed to increased muscle mass and those attributed to fat (Tascilar et al., 2011; Yilmaz et al., 2012). It is important to understand that although BMI and body fat percentage often change simultaneously, a considerable proportion of individuals of certain ethnicities may have a normal BMI but a high body fat content (Kapoor et al., 2019; Kryst et al., 2019). This phenotype is known as 'normal weight obesity' and is commonly described in individuals of Asian descent. The prevalence of normal-weight obesity varies widely among different populations, with the highest prevalence found in Korean women (Kapoor et al., 2019; Kim et al., 2014; Franco et al., 2016). At this point it may be useful to use other available methods to estimate body fat content besides BMI. Two methods most widely used in clinical practice in countries like India are DEXA scanning and BIA (Kapoor et al., 2019). In a study (Yilmaz et al., 2012) comparing the methods used in the assessment of obesity in Turkish children, BMI measurement is recommended as a useful, easily performed, and reproducible method. Moreover, in this study, it was observed that BIA may prevent the false diagnosis of obesity, which is possible when using BMI measurement alone, particularly in boys during the pubertal term. Therefore, although there is a good correlation between body fat percentage and BMI, the limitations of BMI measurements in terms of ethnicity and gender should be considered. It is recommended that researchers consider the use of these indicators not individually, but in conjunction with each other (Kapoor et al., 2019).

The limitation of this study was the cross-sectional design of the DHS dataset which limits the capacity to infer causality. This requires further research using longitudinal data. Another limitation of the present study included a lack of data on nutritional status and physical activity, which 
are important components in the prediction of overweight status due to the unavailability of variables in the dataset.

Despite these limitations, it is necessary to underline the strengths of the study. Firstly, the DHS data produce a nationally representative prediction of overweight among children in Turkey using appropriate weighting techniques. For the first time in this study, the prevalence of overweight in 25 years and the trend of pre-school children in Turkey were examined. As TDHS is repeated every 5 years, all children born from 1988 to 2013 were represented. Secondly, recent studies show that the choice of growth standard is important for identifying the onset of becoming overweight in children (Evellen Vandijk and Inni, 2009). In this study, Z scores were revised according to the recent WHO Child Growth Standards, which is the current reference system instead of NCHS references. Consequently, globally comparable overweight/obesity prevalence was obtained. Thirdly, factors associated with overweight were examined by multilevel analysis, which is appropriate to the nature of the data set.

In conclusion, the rise in overweight/obesity in preschool children since the early 2000s has been dramatic in Turkey. This result points to the need for urgent and effective interventions to reverse worsening trends. Multilevel analyses indicate a relationship between birth weight and overweight gradient, so, affected children should be assessed for becoming overweight in routine health follow-ups from birth. The strong relationship between overweight and stunting indicates the need to clarify the risk factors for the double burden. This study pointed to the relationship between overweight in preschool children and family structures as an important finding. There is a need to focus on identifying potential challenges in single-parent families to help improve family-based interventions. In this study, besides the characteristics of the child such as age and sex, some factors belonging to the household and the parents were found to be associated with being overweight. These findings indicate that interventions that target reducing childhood overweight/obesity should be handled with parents and communities as well as children. The first thing to do for proper intervention programs is to monitor the trend of overweight. Findings are expected to contribute to the plan of prevention strategies to address the rising burden of preschool children's overweight/obesity and its considerable morbidity and mortality in adulthood in Turkey.

Acknowledgments. The authors would like to thank the anonymous reviewers and editor of this journal for their comments, which considerably improved the manuscript. The authors also acknowledge the Hacettepe University Institute of Population Studies and all women and children who participated in TDHS. The authors would also like to thank Ali Görçin and Veene Sulaivany for English language editing.

Ethical Approval. The authors assert that all procedures contributing to this work comply with the ethical standards of the relevant national and institutional committees on human experimentation and with the Helsinki Declaration of 1975, as revised in 2008 .

Conflicts of Interest. The authors have no conflicts of interest to declare.

Funding. This research received no specific grant from any funding agency, commercial entity, or not-for-profit organization.

\section{References}

Armoon B and Karimy M (2019) Epidemiology of childhood overweight, obesity and their related factors in a sample of preschool children from Central Iran. BMC Pediatrics 19(1), 1-8. doi: 10.1186/s12887-019-1540-5

Augustine JM and Kimbro RT (2017) Associations and intervening mechanisms between family structure and young children's obesity. Journal of Family Issues 38(16), 2277-2302. doi: 10.1177/0192513x15621344

Benedict L, Hong SA, Winichagoon P, Tejativaddhana P and Kasemsup V (2020) Double burden of malnutrition and its association with infant and young child feeding practices among children under-five in Thailand. Public Health Nutrition, 1-8. doi: 10.1017/S1368980020003304

Birch LL and Davison KK (2001) Family environmental factors influencing the developing behavioral controls of food intake and childhood overweight. Pediatric Clinics of North America 48(4), 893-907. doi: 10.1016/s0031-3955(05)70347-3 
Brophy S, Cooksey R, Gravenor MB, Mistry R, Thomas N, Lyons RA and Williams R (2009) Risk factors for childhood obesity at age 5: analysis of the millennium cohort study. BMC Public Health 9(1), 1-7. doi: 10.1186/1471-2458-9-467

Byrne LK, Cook KE, Skouteris H and Do M (2011) Parental status and childhood obesity in Australia. International Journal of Pediatric Obesity 6(5-6), 415-418. doi: 10.3109/17477166.2011.598938

Chen AY and Escarce JJ (2010) Peer reviewed: Family structure and childhood obesity, early childhood longitudinal studykindergarten cohort. Preventing Chronic Disease 7(3).

Chen J, Chen W, Zeng G and Li G (2016) Secular trends in growth and nutritional outcomes of children under five years old in Xiamen, China. International Journal of Environmental Research and Public Health 13(11), 1104. doi: 10.3390/ ijerph13111104

Danielzik S, Czerwinski-Mast M, Langnäse K, Dilba B and Müller M (2004) Parental overweight, socioeconomic status and high birth weight are the major determinants of overweight and obesity in 5-7 y-old children: baseline data of the Kiel Obesity Prevention Study (KOPS). International Journal of Obesity 28(11), 1494-1502. doi: 10.1038/sj.ijo.0802756

De Onis M and Blössner M (2000) Prevalence and trends of overweight among preschool children in developing countries. The American journal of clinical nutrition 72(4), 1032-1039. doi: 10.1093/ajcn/72.4.1032

De Onis M, Blössner M and Borghi E (2010) Global prevalence and trends of overweight and obesity among preschool children. The American Journal of Clinical Nutrition 92(5), 1257-1264. doi: 10.3945/ajcn.2010.29786

De Onis M, Onyango AW, Borghi E, Garza C, Yang H and WHO Multicentre Growth Reference Study Group (2006) Comparison of the World Health Organization (WHO) Child Growth Standards and the National Center for Health Statistics/WHO international growth reference: implications for child health programmes. Public Health Nutrition 9(7), 942-947. doi: 10.1017/phn20062005

Deckelbaum RJ and Williams CL (2001) Childhood obesity: the health issue. Obesity Research 9(S11), 239S-243S. doi: 10. 1038/oby.2001.125

Duncan C, Jones K and Moon G (1998) Context, composition and heterogeneity: using multilevel models in health research. Social Science \& Medicine 46(1), 97-117. doi: 10.1016/s0277-9536(97)00148-2

Duriancik DM and Goff CR (2019) Children of single-parent households are at a higher risk of obesity: A systematic review. Journal of Child Health Care 23(3), 358-369. doi: 10.1177/1367493519852463

El-Gamal FM, Babader R, Al-Shaikh M, Al-Harbi A, Al-Kaf J and Al-Kaf W (2020) Study determinants of increased Z-Score of Body Mass Index in preschool-age children. BMC Research Notes 13, 1-5. doi: 10.1186/s13104-020-05026-0

Evellen Vandijk C and Innis SM (2009) Growth-curve standards and the assessment of early excess weight gain in infancy. Pediatrics 123(1), 102-108. doi: 10.1542/peds.2007-3382

Franco LP, Morais CC and Cominetti C (2016) Normal-weight obesity syndrome: diagnosis, prevalence, and clinical implications. Nutrition Reviews 74(9), 558-570. doi: 10.1093/nutrit/nuw019.

Franks PW and Ling C (2010) Epigenetics and obesity: the devil is in the details. BMC Medicine 8(1), 1-5. doi: 10.1186/17417015-8-88

Gewa CA (2010) Childhood overweight and obesity among Kenyan pre-school children: association with maternal and early child nutritional factors. Public Health Nutrition 13(4), 496-503. doi: 10.1017/s136898000999187x

Hamill PV, Drizd TA, Johnson CL, Reed RB and Roche AF (1977) NCHS growth curves for children birth-18 years. Department of Health Education and Welfare Washington DC. URL: https://apps.dtic.mil/sti/pdfs/ADA433981.pdf (accessed 11th September 2020)

Han JC, Lawlor DA and Kimm SY (2010) Childhood obesity. The Lancet 375(9727), 1737-1748. doi: 10.1016/S01406736(10)60171-7

Hediger ML, Overpeck MD, Kuczmarski RJ and Ruan WJ (2001) Association between infant breastfeeding and overweight in young children. JAMA 285(19), 2453-2460. doi: 10.1001/jama.285.19.2453

HIPS (2014) 2013 Turkey Demographic and Health Survey Hacettepe University Institute of Population Studies (HIPS), T.R. Ministry of Development and TÜBİTAK, Ankara, Turkey.

HIPS (2020) Demographic and Health Survey Series. Hacettepe University Institute of Population Studies (HIPS), T.R. Ministry of Development and TÜBİTAK, Ankara, Turkey. URL: http://www.hips.hacettepe.edu.tr/eng/population_ survey.shtml (accessed 12th January 2021).

Hoffman DJ, Roberts SB, Verreschi I, Martins PA, De Nascimento C, Tucker KL and Sawaya AL (2000a) Regulation of energy intake may be impaired in nutritionally stunted children from the shantytowns of Sao Paulo, Brazil. The Journal of Nutrition 130(9), 2265-2270. doi: 10.1093/jn/130.9.2265

Hoffman DJ, Sawaya AL, Verreschi I, Tucker KL and Roberts SB (2000b) Why are nutritionally stunted children at increased risk of obesity? Studies of metabolic rate and fat oxidation in shantytown children from Sao Paulo, Brazil. The American Journal of Clinical Nutrition 72(3), 702-707. doi: 10.1093/ajcn/72.3.702

Huang TT-K and Glass TA (2008) Transforming research strategies for understanding and preventing obesity. JAMA 300(15), 1811-1813. doi: 10.1001/jama.300.15.1811

Huffman FG, Kanikireddy S and Patel M (2010) Parenthood-a contributing factor to childhood obesity. International Journal of Environmental Research and Public Health 7(7), 2800-2810. doi: 10.3390/ijerph7072800

ICF International (2012) MEASURE DHS Biomarker Field Manual. ICF International, Calverton, Maryland, U.S.A. 
ICF International (2018) Demographic and Health Surveys Standard Recode Manual for DHS 7. The Demographic and Health Surveys Program. Rockville, Maryland, U.S.A.

Irvin K, Fahim F, Alshehri S and Kitsantas P (2018) Family structure and children's unmet health-care needs. Journal of Child Health Care 22(1), 57-67. doi: 10.1177/1367493517748372

Jouret B, Ahluwalia N, Cristini C, Dupuy M, Nègre-Pages L, Grandjean H and Tauber M (2007) Factors associated with overweight in preschool-age children in southwestern France. The American Journal of Clinical Nutrition 85(6), 1643-1649. doi: 10.1093/ajcn/85.6.1643

Kapoor N, Furler J, Paul TV, Thomas N and Oldenburg B (2019) The BMI-adiposity conundrum in South Asian populations: need for further research. Journal of Biosocial Science 51(4), 619-621. doi: 10.1017/S0021932019000166

Kim MK, Han K, Kwon HS, Song KH, Yim HW, Lee WC and Park YM (2014) Normal weight obesity in Korean adults. Clinical Endocrinology 80(2), 214-220. doi: 10.1111/cen.12162.

Kondolot M, Balcı E, Ozturk A, Mazıcioglu MM, Hatipoglu N, Kurtoglu S and Ustunbas HB (2011). Body mass index percentiles for Turkish children aged 0-84 months. Annals of Human Biology 38(6), 676-680. doi: 10.3109/03014460.2011. 605800

Kryst L, Żegleń M, Wronka I, Woronkowicz A, Bilińska-Pawlak I, Das R, Saha R, Das S and Dasgupta P (2019) Anthropometric variations in different BMI and adiposity levels among children, adolescents and young adults in Kolkata, India. Journal of Biosocial Science 51(4), 603-618. doi: 10.1017/S0021932018000354.

Kurspahić-Mujčić A and Mujčić A (2020) Factors associated with overweight and obesity in preschool children. Medicinski Glasnik (Zenica) 17(2), 538-543. doi: 10.17392/1175-20

Leddy MA, Power ML and Schulkin J (2008) The impact of maternal obesity on maternal and fetal health. Reviews in Obstetrics \& Gynecology 1(4), 170.

Liu W, Liu W, Lin R, Li B, Pallan M, Cheng K and Adab P (2016) Socioeconomic determinants of childhood obesity among primary school children in Guangzhou, China. BMC Public Health 16(1), 1-8. doi: 10.1186/s12889-016-3171-1

Lobstein T, Baur L and Uauy R (2004) Obesity in children and young people: a crisis in public health. Obesity Reviews 5, 4-85. doi: 10.1111/j.1467-789X.2004.00133.x

Mamabolo RL, Alberts M, Steyn NP, Delemarre-Van De Waal HA and Levitt NS (2005) Prevalence and determinants of stunting and overweight in 3-year-old black South African children residing in the Central Region of Limpopo Province, South Africa. Public Health Nutrition 8(5), 501-508. doi: 10.1079/phn2005786

Marriott BM, Campbell L, Hirsch E and Wilson D (2007). Preliminary data from demographic and health surveys on infant feeding in 20 developing countries. The Journal of Nutrition 137(2), 518-523. doi: 10.1093/jn/137.2.518s

Mccormick DP, Sarpong K, Jordan L, Ray LA and Jain S (2010) Infant obesity: are we ready to make this diagnosis? The Journal of Pediatrics 157(1), 15-19. doi: 10.1016/j.jpeds.2010.01.028

Mohsena M, Goto R and Mascie-Taylor CN (2016) Maternal nutritional status (as measured by height, weight and BMI) in Bangladesh: trends and socio-economic association over the period 1996 to 2007. Public Health Nutrition 19(8), 1438-1445. doi: 10.1017/s1368980015002839

MOH/HUBDB/NH (2014) Turkey Nutrition and Health Survey (TNHS)-2010 (In Turkish). Ministry of Health, Hacettepe University Faculty of Health Sciences Department of Nutrition and Dietetics and Numune Education and Reasearch Hospital (MOH/HUBDB/NH), Republic of Turkey Ministry of Health publications: 931, Ankara, Turkey.

Ogden CL, Carroll MD, Kit BK and Flegal KM (2012) Prevalence of obesity and trends in body mass index among US children and adolescents, 1999-2010. JAMA 307(5), 483-490. doi: 10.1001/jama.2012.40

Oliveria SA, Ellison RC, Moore LL, Gillman MW, Garrahie EJ and Singer MR (1992) Parent-child relationships in nutrient intake: the Framingham Children's Study. The American Journal of Clinical Nutrition 56(3), 593-598. doi: 10.1093/ajcn/56. 3.593

Oppitz IN, Cesar JA and Neumann NA (2014) Overweight among children under five years of age in municipalities of the semiarid region. Revista Brasileira de Epidemiologia 17(4), 860-872. doi: 10.1590/1809-4503201400040006

Pérez LM, García K and Herrera R (2013) Psychological, behavioral and familial factors in obese Cuban children and adolescents. MEDICC review 15, 24-28.

Pérusse L and Bouchard C (2000) Gene-diet interactions in obesity. The American Journal of Clinical Nutrition 72(5), 1285s-1290s. doi: 10.1093/ajcn/72.5.1285s

Portela DS, Vieira TO, Matos SM, De Oliveira NF and Vieira GO (2015) Maternal obesity, environmental factors, cesarean delivery and breastfeeding as determinants of overweight and obesity in children: results from a cohort. BMC Pregnancy and Childbirth 15(1), 1-10. doi: 10.1186/s12884-015-0518-

Qiao Y, Ma J, Wang Y, Li W, Katzmarzyk PT, Chaput J-P, Fogelholm M, Johnson WD, Kuriyan R and Kurpad A (2015) Birth weight and childhood obesity: a 12-country study. International Journal of Obesity Supplements 5(2), S74-S79. doi: 10.1038/ijosup.2015.23

Rasbash J, Steele F, Browne W, Goldstein H and Charlton C (2013). A user's guide to MLwiN. Centre for Multilevel Modelling: University of Bristol. URL: http://www.bristol.ac.uk/cmm/media/software/mlwin/downloads/manuals/3-00/ manual-print.pdf (accessed 11th September 2020) 
Raudenbush SW and Bryk AS (2002) Hierarchical linear models: Applications and data analysis methods. SAGE Publications, U.S.A.

Reilly JJ, Armstrong J, Dorosty AR, Emmett PM, Ness A, Rogers I, Steer C and Sherriff A (2005) Early life risk factors for obesity in childhood: cohort study. BMJ 330(7504), 1357. doi: $10.1136 / \mathrm{bmj} .38470 .670903 . \mathrm{e} 0$

Rooney BL, Mathiason MA and Schauberger CW (2011) Predictors of obesity in childhood, adolescence, and adulthood in a birth cohort. Maternal and Child Health Journal 15(8), 1166-1175. doi: 10.1007/s10995-010-0689-1

Rugholm S, Baker JL, Olsen LW, Schack-Nielsen L, Bua J and Sørensen TI (2005) Stability of the association between birth weight and childhood overweight during the development of the obesity epidemic. Obesity Research 13(12), 2187-2194. doi: $10.1038 /$ oby.2005.271

Ruiz M, Goldblatt P, Morrison J, Porta D, Forastiere F, Hryhorczuk D, Antipkin Y, Saurel-Cubizolles MJ, Lioret S and Vrijheid M (2016) Impact of low maternal education on early childhood overweight and obesity in Europe. Paediatric and Perinatal Epidemiology 30(3), 274-284. doi: 10.1111/ppe.12285

Rutstein SO and Kiersten J (2004) The DHS Wealth Index. DHS Comparative Reports No. 6. Calverton, Maryland, U.S.A.

Sahoo K, Sahoo B, Choudhury AK, Sofi NY, Kumar R and Bhadoria AS (2015) Childhood obesity: causes and consequences. Journal of Family Medicine and Primary Care 4(2), 187. doi: 10.4103/2249-4863.154628

Said-Mohamed R, Bernard JY, Ndzana A-C and Pasquet P (2012) Is overweight in stunted preschool children in Cameroon related to reductions in fat oxidation, resting energy expenditure and physical activity? PloS One 7(6), e39007. doi: 10.1371/ journal.pone.0039007

Santas F and Santas G (2018) Prevalence of pre-school children for overweight/obesity in Turkey. World Journal of Pediatrics 14(1), 77-83. doi: 10.1007/s12519-017-0103-9

Sawaya AL, Grillo LP, Verreschi I, Carlos Da Silva A and Roberts SB (1998) Mild stunting is associated with higher susceptibility to the effects of high fat diets: studies in a shantytown population in Sao Paulo, Brazil. The Journal of Nutrition 128(2), 415S-420S. doi: 10.1093/jn/128.2.415s

Schellong K, Schulz S, Harder T and Plagemann A (2012) Birth weight and long-term overweight risk: systematic review and a meta-analysis including 643,902 persons from 66 studies and 26 countries globally. PloS One 7(10), e47776. doi: 10.1371/ journal.pone.0047776

Schmeer KK (2012) Family structure and obesity in early childhood. Social Science Research 41(4), 820-832. doi: 10.1016/j. ssresearch.2012.01.007

Sisson SB, Sheffield-Morris A, Spicer P, Lora K and Latorre C (2014) Influence of family structure on obesogenic behaviors and placement of bedroom TVs of American children: National Survey of Children's Health 2007. Preventive Medicine 61, 48-53. doi: 10.1016/j.ypmed.2014.01.010

Sorrie MB, Yesuf ME and Gebremichael TG (2017) Overweight/Obesity and associated factors among preschool children in Gondar City, Northwest Ethiopia: A cross-sectional study. PloS One 12(8), e0182511. doi: 10.1371/journal.pone.0182511

Steyn N, Labadarios D, Maunder E, Nel J, Lombard C and Directors of the National Food Consumption Survey (2005) Secondary anthropometric data analysis of the National Food Consumption Survey in South Africa: the double burden. Nutrition 21(1), 4-13. doi: 10.1016/j.nut.2004.09.003

Tascilar ME, Bilir P, Akinci A, Kose K, Akcora D, Inceoglu D and Fitoz SÖ (2011) The effect of gonadotropin-releasing hormone analog treatment (leuprolide) on body fat distribution in idiopathic central precocious puberty. The Turkish Journal of Pediatrics 53(1), 27-33.

Tchoubi S, Sobngwi-Tambekou J, Noubiap JJN, Asangbeh SL, Nkoum BA and Sobngwi E (2015) Prevalence and risk factors of overweight and obesity among children aged 6-59 months in Cameroon: a multistage, stratified cluster sampling nationwide survey. PloS One 10(12), e0143215. doi: 10.1371/journal.pone.0143215

Tsiros MD, Olds T, Buckley JD, Grimshaw P, Brennan L, Walkley J, Hills AP, Howe P and Coates AM (2009) Healthrelated quality of life in obese children and adolescents. International Journal of Obesity 33(4), 387-400. doi: 10.1038/ijo. 2009.42

Tzioumis E and Adair LS (2014) Childhood dual burden of under-and overnutrition in low-and middle-income countries: a critical review. Food and Nutrition Bulletin 35(2), 230-243. doi: 10.1177/156482651403500210

Ucar SK, Dizdarer C, Darcan S, Korkmaz S, Ergudenler Y, Asilsoy S, Bilgili G, Ozcan T, Simsek DG and Coker M (2009) Prevalence of obesity and overweight among children in Izmir, Turkey: effects of nutritional and socio-economic factors. Obesity and Metabolism 5(3/4), 99-106.

United Nations (2015) Sustainable Development Goals. URL: https://sdgs.un.org/goals (accessed 4th December 2020).

WHO Multicentre Growth Reference Study Group and De Onis M (2006) WHO Child Growth Standards based on length/ height, weight and age. Acta Paediatrica 95, 76-85. doi: 10.1111/j.1651-2227.2006.tb02378.x

WHO (2006) A Macro/Programme for Calculating the Z-scores and Prevalences for DHS Individual Flat Files. World Health Organization Department of Nutrition for Health and Development, Geneva.

WHO (2008). Training Course on Child Growth Assessment. World Health Organization, Geneva. URL: https://www.who.int/ childgrowth/training/module_c_interpreting_indicators.pdf?ua=1 (accessed 14th October 2020)

WHO (2010). Anthro for personal computers, version 3.1, 2010: Software for assessing growth and development of the world's children. World Health Organization, Geneva. 
WHO (2013) Resolutions and decisions, sixty-sixth World Health Assembly. World Health Organization, Geneva.

WHO (2016) World Health Statistics 2016: Monitoring Health for the SDGs. World Health Organization, Geneva.

WHO (2019a) Child growth standards, WHO Anthro Survey Analyser and other tools. World Health Organization, Geneva.

URL: https://www.who.int/childgrowth/software/en/ (accessed 14th October 2020)

WHO (2019b) Child growth standards-Q. How different are the new standards from the old growth charts? World Health Organization, Geneva. URL: https://www.who.int/childgrowth/faqs/how_different/en/ (accessed 14th October 2020)

WHO (2020a) Obesity. World Health Organization, Geneva. URL: https://www.who.int/health-topics/obesity\#tab=tab_2 (accessed 4th December 2020).

WHO (2020b) Obesity and overweight. World Health Organization, Geneva. URL: https://www.who.int/en/news-room/factsheets/detail/obesity-and-overweight (accessed 4th December 2020).

Wang X, Höjer B, Guo S, Luo S, Zhou W and Wang Y (2009) Stunting and 'overweight'in the WHO Child Growth Standards-malnutrition among children in a poor area of China. Public Health Nutrition 12(11), 1991-1998. doi: 10. $1017 /$ s1368980009990796

Williams J, Wake M, Hesketh K, Maher E and Waters E (2005) Health-related quality of life of overweight and obese children. JAMA 293(1), 70-76. doi: 10.1016/j.accreview.2005.04.008

Yilmaz D, Inan G, Karakas S, Buyukozturk-Karul A and Sonmez F (2012) Obesity and its diagnostic methods in Turkish children. The Eurasian Journal of Medicine 44(2), 94-98. doi: 10.5152/eajm.2012.22.

Yelick A (2017) The effects of family structure on consumption and exercise patterns for adolescent youth. Child and Adolescent Social Work Journal 34(4), 381-395. doi: 10.1007/s10560-016-0468-y

Yu ZB, Han SP, Zhu GZ, Zhu C, Wang XJ, Cao XG and Guo XR (2011) Birth weight and subsequent risk of obesity: a systematic review and meta-analysis. Obesity Reviews 12(7), 525-542. doi: 10.1111/j.1467-789x.2011.00867.x

Cite this article: Görçin Karaketir Ş, Lüleci NE, Eryurt MA, Emecen AN, Haklıdır M, and Hıdıroğlu S (2023). Overweight and obesity in preschool children in Turkey: A multilevel analysis. Journal of Biosocial Science 55, 344-366. https://doi.org/ $10.1017 /$ S0021932022000025 